\title{
A JND-scale/category-scale convergence in taste
}

\author{
ROBERT L. MCBRIDE \\ Commonwealth Scientific and Industrial Research Organization, Division of Food Research \\ Sydney, Australia \\ and Macquarie University, School of Behavioural Sciences \\ Sydney, Australia
}

\begin{abstract}
The Weber fraction for the sweetness of sucrose was determined at six concentrations. The results provided good support for Weber's law except for deviation near threshold, a finding consistent with previous work. Consequently, the JND scale approximated to Fechner's law. The psychophysical function for sucrose sweetness was also obtained by category rating, with precautions taken to preclude methodological bias. This function was likewise found to conform to Fechner's law, suggesting a JND-scale/category-scale convergence. This convergence was further supported by experiments with the taste stimuli citric acid (acid/sour), sodium chloride (salty), and caffeine (bitter), which showed that the indirectly derived JND scale provides the same measure of taste intensity as the scale obtained directly by category rating.
\end{abstract}

As noted by Birnbaum (1980), perhaps the greatest puzzle in psychophysics has been the failure of different operations to provide a single scale of sensation. In fact, it has commonly been claimed that there are three different types of sensory scalediscriminability (JND), category (partition), and magnitude (ratio) (e.g., see Laming, 1973, p. 38; Stevens, 1961, 1975, p. 228; Stevens \& Stone, 1959).

Recently, however, it has been suggested that two of these scale types may, in fact, provide the same scale of sensation (McBride, 1983, Note 1). When the Weber fraction values for loudness determined by Jesteadt, Wier, and Green (1977) are cumulated in the Fechnerian tradition (Luce \& Edwards, 1958), the resulting JND scale is very similar to the "pure" category scales of loudness reported by Eisler (1962) and Stevens (1975, Figure 50). This JND scale is also in close agreement with loudness scales derived by bisection (Carterette \& Anderson, 1979), equisection (the lambda scale, Garner, 1954), and nonmetric methods (Parker \& Schneider, 1974). The aim of this paper is to explore the relationship between JND and category scales in the taste modality.

Despite its long history in psychophysics, the JND (or DL) scale has been used only sporadically. This is especially true of the taste modality for which, as noted by Meiselman (1972), there has been virtually no indirect scaling. The results of early work on differential sensitivity are incomplete and often

I thank John Best for assistance with the statistical analyses. Requests for reprints should be sent to R. L. McBride, CSIRO Division of Food Research, P. O. Box 52, North Ryde, N.S.W., 2113, Australia. contradictory (e.g., see Bujas, 1937). The first systematic study appears to be that of Schutz and Pilgrim (1957a), who determined Weber fraction values for the taste stimuli sucrose (sweet), citric acid (sour/acid), sodium chloride (salty), and caffeine (bitter) over a range of concentrations. Overall, Schutz and Pilgrim found reasonable support for Weber's law, which suggests that, if these Weber functions were cumulated, the resulting JND scales would approximate to Fechner's log law.

But Torgerson $(1958$, p. 151) stresses that if the JND scale is to have scientific credibility, then the Weber function from which it is derived must be robust and not affected by the experimental method used to obtain it. In other words, Weber functions that have been obtained by different methods should be determined to within a linear transformation. Schutz and Pilgrim (1957a) used the method of single stimuli (Pfaffmann, 1935) in their investigation. The first experiment in the present study redetermined the Weber function for sucrose by the method of constant stimuli, generally held to be the most sensitive of the classical psychophysical methods (Guilford, 1954, p. 118), and compared it with the function reported by Schutz and Pilgrim.

Some previous work (Schutz \& Pilgrim, 1957b; Vaisey Genser \& Moskowitz, 1977, p. 35) suggests that, like the JND scale, the category scale of sucrose sweetness also approximates to Fechner's law. However, category scaling is prone to methodological bias-range effects, frequency effects, sequential effects (Parducci, 1974; Poulton, 1979). Perhaps the most frequently cited drawback of category scaling in psychophysics is its susceptibility to stimulus spacing bias; that is, the shape of the psycho- 
physical function obtained by category rating is dependent on the spacing of the stimulus intensities (Stevens \& Galanter, 1957).

Nevertheless, Pollack (1965) and Stevens (1975, p. 141) have described an iterative procedure for eliminating the bias due to arbitrary stimulus spacing, a procedure investigated theoretically elsewhere (Anderson, 1975). Essentially, this procedure consists of first presenting subjects with an arbitrarily spaced set of stimuli. The psychophysical scale obtained from this initial presentation then determines the stimulus spacing for the next iteration, and so on, until the scales dictated by consecutive iterations converge; that is, the scale last obtained suggests the same spacing as that used to obtain it. Such a scale is then said to be in pure, or unbiased, form.

In addition to the JND determination, the first experiment in the present study involved deriving the psychophysical function for sucrose sweetness by category rating. Both geometric and arithmetic stimulus spacing were used, and the iterative technique was applied where necessary.

With regard to range bias (or centering bias), Poulton $(1977,1979)$ showed that, for a sensory scale to be free from such influence, the overall mean response score should correspond to the midpoint of the rating scale. This requirement was also checked in the present experiment.

\section{EXPERIMENT 1}

\section{Method}

\section{Determination of the Sucrose Weber Function}

Subjects. The 20 subjects (age range 25-58 years) were all employees of the CSIRO Food Research Laboratory and participated voluntarily. Prior to the experiment, most were familiar with the sensory evaluation of food, but few were experienced in psychophysical tasks. Overall, there were approximately equal numbers of responses from men and women.

Stimuli. Stimuli consisted of reagent-grade sucrose in distilled water. There were six concentration levels (standards)$.025, .050, .100, .200, .300$, and $.500 \mathrm{M}$. These levels approximately match those of Schutz and Pilgrim (1957a). There were six arithmetically spaced comparison stimuli at each level. At the lowest level $(.025 \mathrm{M})$, the comparison stimuli were $.50, .70$, $.90,1.10,1.30$, and 1.50 times the standard; for the upper five levels, preliminary testing suggested greater sensitivity, and the comparison stimuli were therefore $.70, .82, .94,1.06,1.18$, and 1.30 times the standard. Stimulus temperature was approximately $20^{\circ} \mathrm{C}$, and volume was $30 \mathrm{ml}$.

Procedure. The procedure employed was a forced-choice variant of the method of constant stimuli in which the standard is not disclosed (cf. Harrison \& Harrison, 1951). The design was similar to that of Lundgren, Pangborn, Barylko-Pikielna, and Daget (1976), but fewer stimuli were presented at each testing session to minimize disruption to subjects' work routine.

There were 60 experimental sessions, 10 sessions per concentration level; 2 sessions were run per day. Only one concentration level was assessed per day; the order of assessment of levels across days was random. At each session, the subjects were presented with three coded pairs of solutions, each pair consisting of the standard and a comparison stimulus. Order of tasting both between and within pairs was randomized. The subjects were instructed to taste and expectorate the solutionis within a pair (the "sip and spit" technique), and to identify the sweeter solution. Thorough rinsing with distilled water was mandatory between pairs. The 20 sets of three pairs (one set per subject) presented at each session comprised all possible combinations of the six comparison stimuli taken three at a time. The 60 responses from each session thus provided an overall total of 3,600 responses, 100 responses for each of the 36 standard-comparison pairs.

Data treatment. The percentage of "sweeter" responses was calculated for each of the six comparison stimuli at each concentration level. These data were then analyzed in two different ways. In the first, and more traditional, analysis, the percentages were converted to normal deviates and fitted against log sucrose concentration (the phi-log-gamma hypothesis, Rubin, 1976; Thurstone, 1928) by the least squares procedure (Guilford, 1954, p. 125). In the second analysis, arc sine transforms of the percentages $\left(\sin ^{-1} \sqrt{p / 100}\right.$, where $p$ is the percentage) were fitted against sucrose concentration by the method of least squares. To facilitate comparison with the Schutz and Pilgrim (1957a) study, the JND was computed in traditional fashion in each case, by halving the difference between the concentrations corresponding to the $75 \%$ and $25 \%$ points on the ordinate. As a variance-stabilizing measure (Box, Hunter, \& Hunter, 1978, p. 132), the arc sine transform allows estimation of the standard errors of the JNDs, and hence the error of the cumulated JND scale. The approximate standard errors were calculated from the formula for the variance of a function (Kendall \& Stuart, 1977, p. 246).

\section{Category Scaling}

Subjects. Twenty-four subjects were used-the 20 from the JND determination and four others drawn from the same pool.

Stimuli. Stimuli were reagent-grade sucrose in distilled water. In Part 1 , the concentrations were $.0625, .1250, .2500$, and $.5000 \mathrm{M}$ (four stimuli, geometric/log spacing); in Part 2, .0625, $.0947, .1436, .2177, .3299$, and $.5000 \mathrm{M}$ (six stimuli, log spacing); and in Part $3, .0625, .2083, .3542$, and $.5000 \mathrm{M}$ (four stimuli, arithmetic spacing). In each case, the stimulus temperature and volume were the same as in the JND determination.

Response scale. The scale comprised 13 categories with five equidistant verbal descriptors: "Extremely sweet" (13), "Very sweet" (10), "Moderately sweet" (7), "Slightly sweet" (4), and "No sweetness at all" (1). As such, it was in accord with Anderson's (1974) suggestion that between 10 and 20 categories is desirable, and also with Bendig and Hughes (1953), who found that verbal anchors increase the amount of information transmitted by a scale. However, stimulus end anchors were not presented, nor were the categories numbered on the subjects' response sheet.

Procedure. Parts 1 and 2 each consisted of two replicate sessions, the replicate sessions within each part being held on consecutive days. Stimuli were presented simultaneously, and subjects were required to taste and expectorate each in the order specified and to rate each sweetness on the 13-point scale. Rinsing with distilled water was mandatory between stimuli. All 24 possible permutations of presentation order were used in Part 1, while in Part 2, order was randomized.

In Part 3, the procedure was identical, except that arithmetic stimulus spacing was used at the first session. The sweetness scale from the initial session suggested revised concentrations for the second session on the following day, and so on, until the sweetness scales converged at the fourth session (Pollack, 1965 , provides details of the methodology).

\section{Results}

\section{The Sucrose Weber Function}

Figure 1 shows the JND estimates obtained by the arc sine transform method plotted against su- 


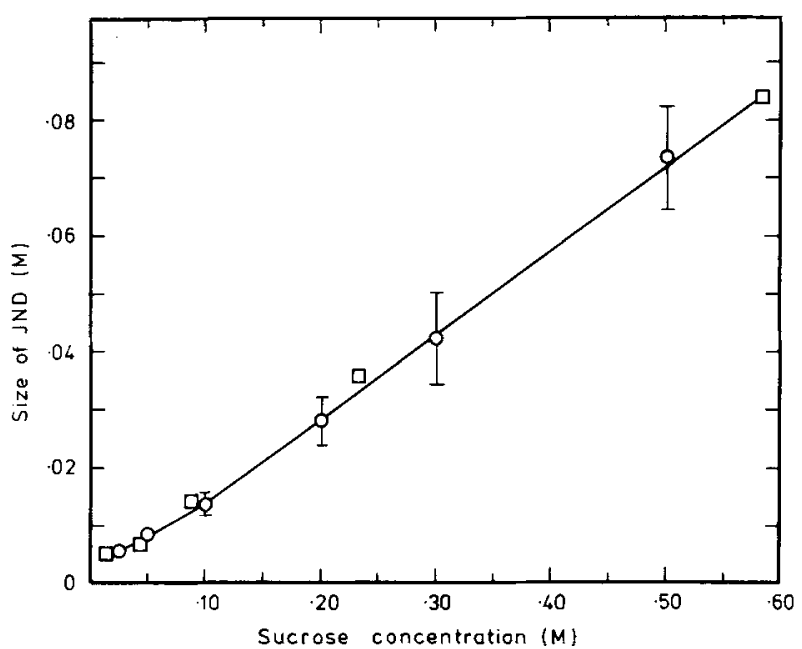

Figure 1. The size of the JND vs. sucrose concentration in molar (M) units. The line of best fit was drawn by eye. Data from the present study (circles, $\pm 2 \mathrm{SE}$; error bars for the two lowest concentrations are within the data points) and those of Schutz and Pilgrim (1957b) (squares) are in close agreement. Except for the low concentrations near threshold, there is good approximation to Weber's law.

crose concentration (circles $\pm 2 \mathrm{SE}$ ). Converting the data to normal deviates and fitting against log concentration, as in the first analysis described, gave an almost identical result. For all but the highest concentration $(.5000 \mathrm{M})$, for which the estimate was discrepant by $4 \%$, the JND values coincided with the circles in Figure 1.

The values of Schutz and Pilgrim (1957a) are shown by the squares in Figure 1. Clearly, Torgerson's linear transformation criterion is satisfied: Despite the many differences between the present study and that of Schutz and Pilgrim (e.g., in psychophysical method, stimulus volume, state of adaptation), correspondence between the two sets of data is sufficiently close to permit representation by a common curve (drawn by eye).

Apart from deviation at low concentration, it is apparent from Figure 1 that the size of the JND is directly proportional to sucrose concentration, as dictated by Weber's law. Weber's law may break down again at very high concentration, as it does for sodium chloride (Holway \& Hurvich, 1937; Schutz \& Pilgrim, 1957a), but this cannot be ascertained using a discrimination paradigm. The viscosity of sucrose solutions increases markedly above $.5000 \mathrm{M}$ : The confounding of viscosity and sweetness would leave the investigator uncertain as to which cue serves in the discrimination (cf. MacLeod, 1952).

\section{JND Scale}

Figure 2 illustrates the JND scale that results when the Weber function of Figure 1 is cumulated in the
Fechnerian tradition. As expected from Figure 1, there is good support for Fechner's law except for the deviation near threshold (taken here as $.015 \mathrm{M}$; cf. Amerine, Pangborn, \& Roessler, 1965, pp. 88, 97; Pfaffman, 1959). The envelope (dotted lines) surrounding the JND scale represents approximately $\pm 2 \mathrm{SE}$. The standard error at any one point on the JND scale was taken as the square root of the sum of the squared standard errors of the JNDs cumulated up to this point (Kendall \& Stuart, p. 246). Here the standard errors lie horizontally on the JND scale; by fiat, there can be no error in the vertical dimension.

\section{Category Scale}

For each replicate in Parts 1 and 2 separately, and for data from the final session in Part 3, category ratings were fitted against $\log$ sucrose concentration by linear regression. There was no significant difference between the estimates of slope $[F(4,566)$ $=1.20]$, indicating good scale reliability.

To facilitate the JND-scale/category-scale comparison, the category ratings from Parts 1 and 2, and from the final session of Part 3, were then fitted against log sucrose concentration by a single linear regression. Next, the category scale was positioned on the right-hand ordinate of Figure 2 so that this regression line would coincide with the JND scale (legitimate, since the category scale is an interval scale). The mean category ratings $( \pm 2 \mathrm{SE})$ from Parts 1, 2, and 3 are given in Figure 2.

The data in Figure 2 support a JND-scale/categoryscale convergence. Most of the mean category ratings

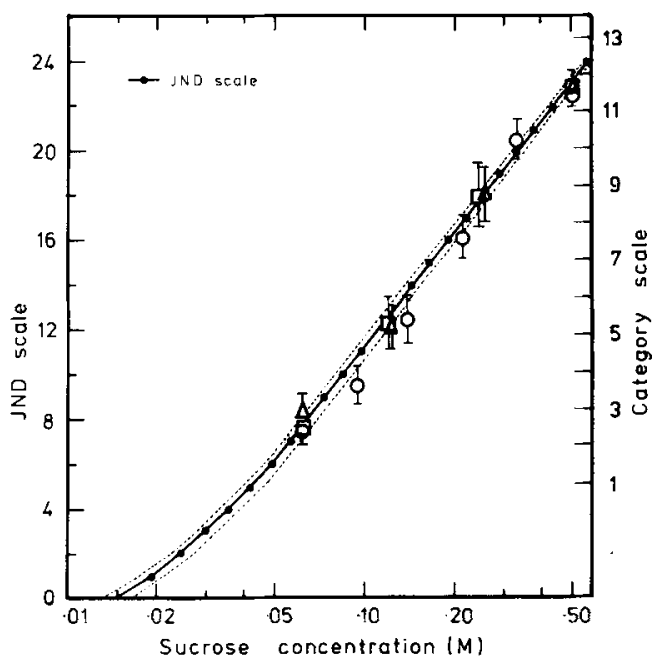

Figure 2. The JND scale (left-hand ordinate) and the category scale (right-hand ordinate) for the sweetness of sucrose over the concentration range $.0625-.5000 \mathrm{M}$. The mean ratings $( \pm 2$ SE) from Parts 1 (triangles), 2 (circles), and 3 (squares) conform to the JND scale, suggesting a JND-seale/category-scale convergence (dotted line represents approximately \pm 2 SE for the JND scale). 
actually lie within the error envelope of the JND scale, conforming to Fechner's law. Moreover, the mean category ratings in each part of this experiment are approximately equidistant on the ordinate, as required of a pure category scale. The mean responses to the final iteration of Part 3 confirm that obtaining the pure category scale straight off, using geometric stimulus spacing (Parts 1 and 2), was not coincidence. Even when arithmetic stimulus spacing is used initially, the iterative procedure dictates geometric spacing for a pure scale.

A check on the 14 mean category ratings in Parts 1-3 showed no evidence of range bias. The overall mean rating of 6.96 corresponds closely to 7 , the scale midpoint. This result is somewhat fortuitous, since the sucrose concentrations used in the category rating experiments were chosen to match those in the JND determinations, and not specifically selected to be exempt from range bias.

\section{EXPERIMENT 2}

Experiment 2 investigates the JND-scale/categoryscale relationship with another three stimuli-citric acid (acid/sour), sodium chloride (salty), and caffeine (bitter). However, in view of the close correspondence between previous and present data established in Experiment 1, Weber functions for these three stimuli were not determined experimentally but were taken from Schutz and Pilgrim (1957a).

\section{Method}

\section{Category Scaling}

Subjects. A panel of 24 subjects was used. Composition of the panel remained constant during testing of a single taste quality, but altered slightly between qualities.

Stimuli. Four concentrations of a reagent-grade chemical in distilled water were used for each taste. For citric acid, the concentrations were $.0010, .0030, .0090$, and $.0270 \mathrm{M}$; for sodium chloride, .0513, .0923,.1641, and .2906 $\mathrm{M}$; and for caffeine, $.0031, .0066, .0144$, and $.0309 \mathrm{M}$. Logarithmic spacing was used in all cases, and the concentration ranges are commensurate with those used by Schutz and Pilgrim (1957a) in their Weber function determinations.

Response scale. Except for the appropriate changes in descriptors (e.g., "Extremely salty," "Extremely bitter"), the response scale was as used in Experiment 1.

Procedure. The procedure was as for Parts 1 and 2 in Experiment 1: There were two replicate sessions for each of the three taste stimuli.

\section{Results}

\section{JND Scales}

The JND scales for citric acid, sodium chloride, and caffeine, generated by cumulating the Weber functions of Schutz and Pilgrim (1957a, Tables 1 and 2), are given in Figures 3, 4, and 5, respectively. Except for the deviation near threshold, there is

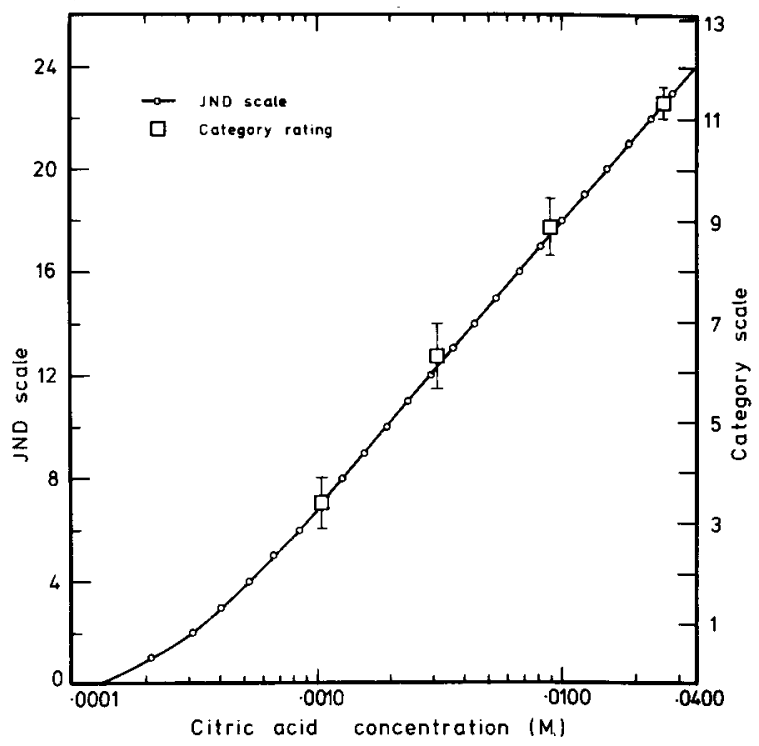

Figure 3. The JND scale (left-hand ordinate) and category scale (right-hand ordinate) for the perceived acidity of citric acid. Mean category ratings ( $\pm 2 \mathrm{SE}$ ) conform to the JND scale.

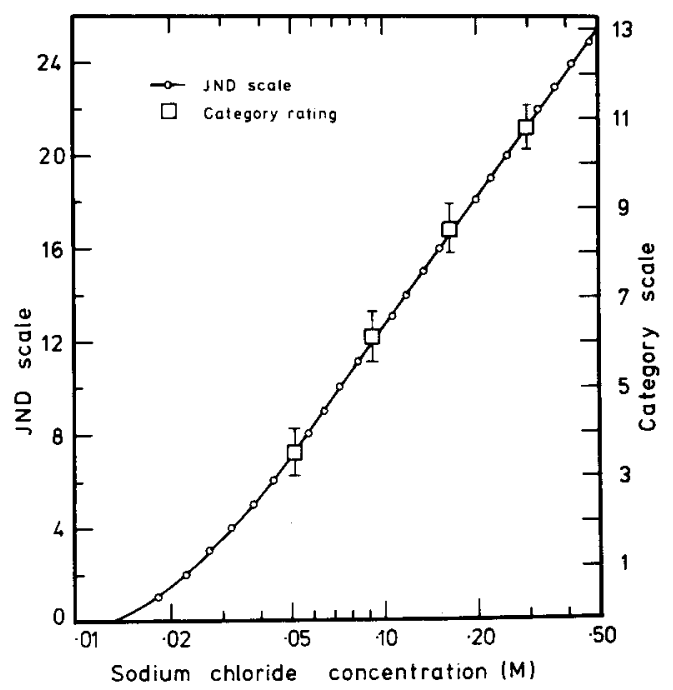

Figure 4. The JND scale (left-hand ordinate) and category scale (right-hand ordinate) for the perceived saltiness of sodium chloride. Mean category ratings ( \pm 2 SE) conform to the JND scale.

once again support for Fechner's law, at least over the stimulus ranges shown.

The threshold value for citric acid was taken as .00014 M (Berg, Filipello, Hinreiner, \& Webb, 1955; Stahl, 1973); for sodium chloride, .0137 M (Pfaffmann, 1959; Stahl, 1973); and for caffeine, .0002 M (Amerine et al., p. 106). There is greater variability in reported caffeine thresholds, possibly due to the greater dif- 


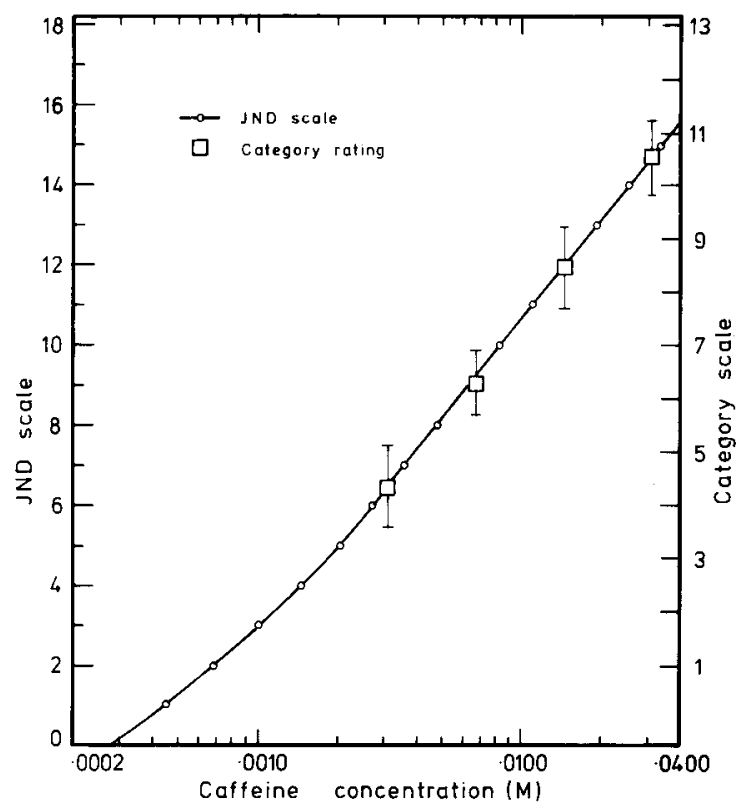

Figure 5. The JND scale (left-hand ordinate) and category scale (right-hand ordinate) for the perceived bitterness of caffeine. Mean category ratings ( \pm 2 SE) conform to the $J N D$ scale.

ficulty in identifying dilute bitter solutions (Amerine et al., p. 107).

\section{Category Scales}

For each of the three taste qualities, category ratings were fitted against log stimulus concentration by linear regression, then the category scales were positioned on the right-hand ordinate, as described in Experiment 1. Figures 3-5 contain the mean category ratings, $\pm 2 \mathrm{SE}$; the error bars are approximately the same length regardless of taste intensity. Clearly, there is further evidence of a JND-scale/ category-scale convergence. Moreover, in all three cases, the mean category ratings are equidistant on the ordinate (pure category scale), and in each case the overall mean rating corresponds approximately to the midpoint of the rating scale (absence of centering bias).

\section{DISCUSSION}

Evidence of a JND-scale/category-scale convergence has fundamental significance for psychophysics, for it indicates that scales derived indirectly in the classical Fechnerian tradition may be no different from scales obtained by direct judgment.

Still, the question of scale validity is pertinent here. With support for the reproducibility of the Weber function from Experiment 1, a discussion of JND-scale validity reduces to a discussion of the validity of Fechner's assumption that all JNDs are subjectively equal (Torgerson, 1958, p. 150). Stevens (1957) rejected Fechner's assumption on the basis of failure of Fechner's law to account for loudness data. It has been shown, however, that failure of Fechner's law in loudness may be explained in terms of failure of Weber's law, and that Fechner's assumption is not necessarily invalid (McBride, 1983). Parker and Schneider (1980) reached the same conclusion in their analysis of loudness data.

With regard to category scaling, the function for sucrose obtained in the present study with a 13-point response scale is very similar to that obtained by Schutz and Pilgrim (1957b) with a 9-point scale: Both functions approximate well to Fechner's law. Also, the response variability of the category ratings in Figures 2-5 is approximately constant irrespective of subjective magnitude, consistent with Fechner's assumption. On the validity question, it should be mentioned that there is now substantial support for category rating as a linear response measure from studies in functional measurement analysis (Anderson, $1970,1972,1974,1981$, pp. 9, 348). This provides further support, albeit indirect, for the equal-interval properties of the JND scale. If JNDs are subjectively equal, then the JND scales in Figures 2-5 are ratio scales; that is, they each have equal-interval properties and a meaningful zero.

Figures 2-4 also provide some evidence for the subjective equality of JNDs between taste continua. For sucrose, citric acid, and sodium chloride, there are approximately 22 JNDs between threshold and a subjective intensity level corresponding to a mean rating of 11 on the category scale. The corresponding number of JNDs for caffeine is lower, at 15. Bujas (1937), using a sucrose/sodium-chloride matching paradigm, also found support for an intercontinuum equivalence in the subjective size of the JND. These findings contrast with recent work on intensity resolution in loudness, which suggests that subjects match stimuli on the basis of their relative positions in the dynamic range, and not on the basis of absolute number of JNDs above threshold (the "proportional JND" explanation; Houtsma, Durlach, \& Braida, 1980).

An interesting aspect of the present data was the absence of stimulus spacing bias. In Part 3 of Experiment 1 , in which arithmetic spacing was used initially, the mean responses to the four stimuli at the first session were, in ascending order, 3.3, 7.9, 11.1, and 11.4. These responses lie very close to the final iterated form of the scale shown in Figure 2. In other words, while the iterative procedure was effective in producing mean responses that are equidistant on the ordinate, the shape of the psychophysical function did not change over the course of the four sessions. It appears, therefore, that, in this instance, subjects were not influenced by 
immediate context and made their judgments of sweetness in an absolute, rather than relative, manner.

Two explanations are possible. First, in the present study there actually was less "context" than in many other psychophysical investigations (e.g., Parducci, 1974); with only four stimuli presented at each session, there is little chance of the stimulus distribution influencing judgment. Second, stimulus end anchors were not used in the present study; the only reference points available to subjects were the verbal descriptors attached to the response scale. Presence of these descriptors may have facilitated the evaluation of each stimulus in terms of absolute sweetness.

Finally, the present findings raise the question of why it is that JND scales and category scales have traditionally been regarded as disparate. Stevens (1961) claimed that, on all prothetic continua, the JND scale approximates a logarithmic function and the category scale assumes a form somewhat less than logarithmic.

First, the assertion that the JND scale is approximately logarithmic is true only to the extent that the empirical Weber function approximates to Weber's law. The deviation from Weber's law at low stimulus intensity occurs on most continua (Holway \& Pratt, 1936). Therefore, if the JND scale is generated from an empirical Weber function and not, as has often been the case, from Weber's law or its linear generalization (e.g., Stevens, 1959, 1961), it will be found to be less than logarithmic. This is particularly true of auditory stimuli (Houtsma et al., 1980).

Second, methodological bias in category rating has not always been given due attention. Although stimulus spacing bias was found not to operate in the present study, it cannot be dismissed. Range effects must also be considered. In the scaling of finger span (Stevens \& Stone, 1959), for example, the category scale used in the JND-scale/categoryscale comparison is not a pure scale: Arithmetic stimulus spacing was used, and the mean responses are not equispaced on the ordinate. This spacing may have flattened the response curve in the manner described by Stevens and Galanter (1957), causing a divergence from the JND scale.

\section{REFERENCE NOTE}

1. McBride, R. L. Psychophysics: Where everything old is new again. Paper presented at the 8th Australian Experimental Psychology Conference, Adelaide, May 1981.

\section{REFERENCES}

Amerine, M. A., Pangborn, R. M., \& Roessler, E. B. Principles of sensory evaluation of food. New York: Academic Press, 1965.
Anderson, N. H. Functional measurement and psychophysical judgment. Psychological Review, 1970, 77, 153-170.

Anderson, N. H. Cross-task validation of functional measurement. Perception \& Psychophysics, 1972, 12, 389-395.

Anderson, N. H. Algebraic models in perception. In E. C. Carterette \& M. P. Friedman (Eds.), Handbook of perception (Vol. 2). New York: Academic Press, 1974.

Anderson, N. H. On the role of context effects in psychophysical judgment. Psychological Review, 1975, 82, 462-482.

Anderson, N. H. Foundations of information integration theory. New York: Academic Press, 1981.

Bendig, A. W., \& Hughes, J. B., II. Effect of amount of verbal anchoring and number of rating-scale categories upon transmitted information. Journal of Experimental Psychology, 1953, 46, 87-90.

Berg, H. W., Filipello, F., Hinreiner, E., \& Webe, A. D. Evaluation of thresholds and minimum difference concentrations for various constituents of wines. I. Water solutions of pure substances. Food Technology, 1955, 9 (1), 23-26.

Birnbaum, M. H. Comparison of two theories of "ratio" and "difference" judgments. Journal of Experimental Psychology: General, 1980, 109, 304-319.

Box, G. E. P., Hunter, W. G., \& Hunter, J. S. Statistics for experimenters. New York: Wiley, 1978.

Bujas, Z. La mesure de la sensibilité differentielle dans la domaine gustatif. Acta Instituti Psychologici, 1937, 2, 1-18.

Cartenette, E. C., \& Anderson, N. H. Bisection of loudness. Perception \& Psychophysics, 1979, 26, 265-280.

EIsLer, H. Empirical test of a model relating magnitude and category scales. Scandinavian Journal of Psychology, 1962, 3, 88-96.

Garner, W. R. A technique and a scale for loudness measurement. Journal of the Acoustical Society of America, 1954, 26, 73-88.

Guilford, J. P. Psychometric methods (2nd ed.). New York: McGraw-Hill, 1954.

Harrison, S., \& Harrison, M. J. A psychophysical method employing a modification of the Muller-Urban weights. Psychological Bulletin, 1951, 48, 249-256.

Holway, A. H., \& Hurvich, L. M. Differential gustatory sensitivity to salt. American Journal of Psychology, 1937, 49, $37-48$.

Holway, A. H., \& Pratt, C. C. The Weber-ratio for intensitive discrimination. Psychological Review, 1936, 43, 322-340.

Houtsma, A. J. M., Durlach, N. I., \& Braida, L. D. Intensity perception XI. Experimental results on the relation of intensity resolution to loudness matching. Journal of the Acoustical Society of America, 1980, 68, 807-813.

Jesteadt, W., Wier, C. C., \& Green, D. M. Intensity discrimination as a function of frequency and sensation level. Journal of the Acoustical Society of America, 1977, 61, 169-177.

Kendall, M., \& Stuart, A. The advanced theory of statistics (Vol. 1, 4th ed.). London: Griffin, 1977.

Laming, D. Mathematical psychology. London: Academic Press, 1973.

LUCE, R. D., \& EDwards, W. The derivation of subjective scales from just noticeable differences. Psychological Review, $1958,65,222-237$.

Lundgren, B., Pangbohn, R. M., Barylko-Pikielna, N., \& DAGET, N. Difference taste thresholds for sucrose in water and in orange juice: An interlaboratory study. Chemical Senses and Flavor, 1976, 2, 157.176.

MAcLeod, S. A construction and attempted validation of sensory sweetness scales. Journal of Experimental Psychology, 1952, 44, 316-323.

McBride, R. L. Psychophysics: Could Fechner's assumption be correct? Australian Journal of Psychology, 1983, 35, 85-88.

Meiselman, H. L. Human taste perception. CRC Critical Reviews in Food Technology, 1972, 3, 89-119.

Parducci, A. Contextual effects: A range-frequency analysis. In 
E. C. Carterette \& M. P. Friedman (Eds.), Handbook of perception (Vol. 2). New York: Academic Press, 1974.

Parker, S., \& Schnetder, B. Nonmetric scaling of loudness and pitch using similarity and difference estimates. Perception \& Psychophysics, 1974, 15, 238-242.

Parker, S., \& Schneider, B. Loudness and loudness discrimination. Perception \& Psychophysics, 1980, 28, 398-406.

Pfaffmann, C. An experimental comparison of the method of single stimuli and the method of constant stimuli in gustation. American Journal of Psychology, 1935, 37, 470-475.

Praffmann, C. The sense of taste. In J. Field (Ed.), Handbook of physiology (Vol. 1). Washington, D.C: American Physiological Society, 1959.

Potlack, I. Iterative techniques for unbiased rating scales. Quarterly Journal of Experimental Psychology, 1965, 17, 139-148.

Poulton, E. C. Quantitative subjective assessments are almost always biased, sometimes completely misleading. British Journal of Psychology, 1977, 68, 409-425.

Poulton, E. C. Models for biases in judging sensory magnitude. Psychological Bulletin, 1979, 86, 777-803.

Rubin, D. C. Frequency of occurrence as a psychophysical continuum: Weber's fraction, Ekman's fraction, range effects, and the phi-gamma hypothesis. Perception \& Psychophysics, 1976, 20, 327-330.

Schutz, H. G., \& Pilgrim, F. J. Differential sensitivity in gustation. Journal of Experimental Psychology, 1957, 54, 41-48. (a)

Schutz, H. G., \& Pilgrim, F. J. Sweetness of various com- pounds and its measurement. Food Research, 1957, 22, 206213. (b)

StAHL, W. H. (Ed.). Compilation of odor and taste threshold values data. Philadelphia: American Society for Testing and Materials, 1973.

Stevens, S. S. On the psychophysical law. Psychological Review, 1957, 64, 153-181.

Stevens, S. S. Tactile vibration: Dynamics of sensory intensity. Journal of Experimental Psychology, 1959, 57, 210-218.

STEvens, S. S. The psychophysics of sensory function. In W. A. Rosenblith (Ed.), Sensory communication. Cambridge, Mass: M.I.T. Press, 1961.

Stevens, S. S. Psychophysics. New York: Wiley, 1975.

Stevens, S. S., \& Galanter, E. H. Ratio scales and category scales for a dozen perceptual continua. Journal of Experimental Psychology, 1957, 54, 377-411.

Stevens, S. S., \& Stone, G. Finger span: Ratio scale, category scale, and JND scale. Journal of Experimental Psychology, 1959, 57, 91-95.

Thurstone, L. L. The phi-gamma hypothesis. Journal of Experimental Psychology, 1928, 11, 293-305.

Togenson, W. S. Theory and methods of scaling. New York: Wiley, 1958.

Vaisey Genser, M., \& Moskowitz, H. R. Sensory response to food. Zurich: Forster, 1977.

(Manuscript received August 23, 1982;

revision accepted for publication February 15, 1983.) 